Article

\title{
Synthesis of Novel Dihydropyridothienopyrimidin-4,9-dione Derivatives
}

\author{
Youngjae Kim ${ }^{1,2}$, Minjoo Kim ${ }^{1,3}$, Mooseong Park ${ }^{1,4}$, Jinsung Tae ${ }^{4}$, Du-Jong Baek ${ }^{3}$, \\ Ki Duk Park ${ }^{1,4}$ and Hyunah Choo ${ }^{1,4, *}$
}

1 Center for Neuro-Medicine, Korea Institute of Science and Technology, Seongbuk-gu, Seoul 136-791, Korea; E-Mails: 213501@kist.re.kr (Y.K.); yonlyk@naver.com (M.K.); h14507@kist.re.kr (M.P.); kdpark@kist.re.kr (K.D.P.)

2 Department of Chemistry, Yonsei University, Seodaemun-gu, Seoul 120-749, Korea

3 Department of Chemistry, College of Natural Sciences, Sangmyung University, Seoul 110-743, Korea; E-Mail: djbaek@smu.ac.kr

4 Department of Biological Chemistry, University of Science and Technology, Youseong-gu, Daejeon 305-350, Korea; E-Mail: jstae@yonsei.ac.kr

* Author to whom correspondence should be addressed; E-Mail: hchoo@kist.re.kr; Tel.: +82-2-958-5157.

Academic Editor: Derek J. McPhee

Received: 27 January 2015 / Accepted: 13 March 2015 / Published: 19 March 2015

\begin{abstract}
A novel molecular scaffold, dihydropyridothienopyrimidin-4,9-dione, was synthesized from benzylamine or $p$-methoxybenzylamine in six steps involving successive ring closure to form a fused ring system composed of dihydropyridone, thiophene and pyrimidone. The pharmacological versatility of the dihydropyridothenopyrimidin-4,9-dione scaffold was demonstrated by inhibitory activity against metabotropic glutamate receptor subtype 1 (mGluR1), which shows that the title compounds can serve as an interesting scaffold for the discovery of potential bioactive molecules for the treatment of human diseases.
\end{abstract}

Keywords: dihydropyridothienopyrimidin-4,9-dione; pyridothienopyrimidine; successive ring formation 


\section{Introduction}

Much attention has been paid to the thienopyrimidine and thienopyridine scaffolds due to their notable biological and pharmacological activities (Figure 1) [1-6]. Thienopyrimidines, like $(R)-N$-(3-chloro-4((3-fluorobenzyl)oxy)phenyl)-6-(pyrrolidin-2-ylethynyl)thieno[3,2- $d]$ pyrimidin-4-amine and 6-(1-benzyl-1H-indol-3-yl)-2-(piperidin-1-ylmethyl)thieno[3,2- $d]$ pyrimidin-4(3H)-one, show potent anticancer activity by inhibition of epidermal growth factor receptor (EGFR) [1] and vascular endothelial growth factor receptor-2 (VEGFR-2) [2], respectively. On the other hand, thienopyridine derivatives such as ticlopidine, clopidogrel, and prasugrel block $\mathrm{P}_{2} \mathrm{Y}_{12}$ receptors and thus inhibit platelet activation and aggregation [3-6].

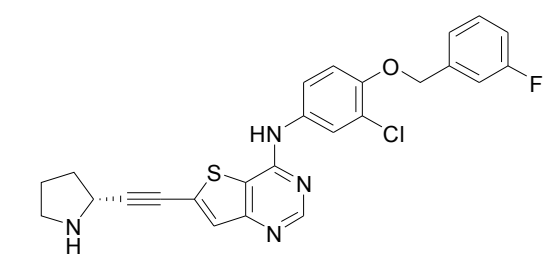

(R)- $N$-(3-Chloro-4-((3-fluorobenzyl)oxy)phenyl)-6(pyrrolidin-2-ylethynyl)thieno[3,2- $d]$ pyrimidin-4-amine

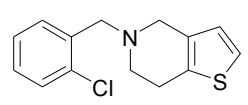

Ticlopidine

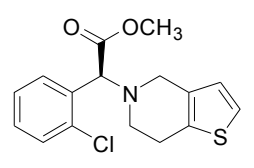

Clopidogrel

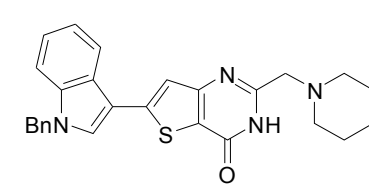

6-(1-Benzyl-1H-indol-3-yl)-2-(piperidin-1ylmethyl)thieno[3,2-d]pyrimidin-4(3H)-one

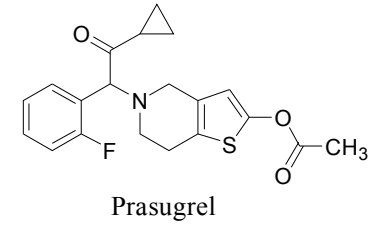

Figure 1. Bioactive thienopyrimidine and thienopyridine derivatives.

Accordingly, combining both the thienopyrimidine and thienopyridine moieties in the same molecular framework (i.e., pyridothienopyrimidine 1, Figure 2) has served as an attractive strategy for designing a novel scaffold with more favorable pharmacological properties [7]. In this study, as a part of our ongoing efforts to synthesize fused tricyclic heterocycles, dihydropyridinothienopyrimidin-4,9dione derivatives 2 were prepared starting from benzylamine or $p$-methoxybenzylamine in six steps involving successive ring formations. Also, to demonstrate the biological and pharmacological versatility, the synthesized compounds were biologically evaluated against metabotropic glutamate receptor subtype 1 (mGluR1) which is one of molecular targets for treatment of neuropathic pain.
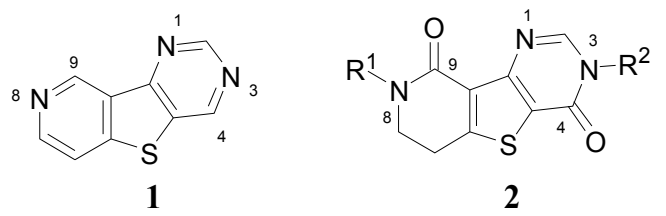

Figure 2. Pyridothienopyrimidine (1) and dihydropyridothienopyrimidin-4,9-diones 2.

\section{Results and Discussion}

To synthesize the target compounds $\mathbf{2}$, the reaction sequence shown in Scheme 1 was used. Benzylamines 3a and $\mathbf{3 b}$ were used as starting materials. Thus, benzylamine 3a or $p$-methoxybenzylamine $3 \mathbf{b}$ were treated with ethyl acrylate in the presence of catalytic $\mathrm{Cu}(\mathrm{OAc})_{2}$ to give 
the corresponding $\beta$-aminoester $4 \mathbf{a}$ or $\mathbf{4 b}$ (47\% or $57 \%$ yield, respectively). The $\beta$-aminoesters $\mathbf{4 a}$ and 4b were coupled with cyanoacetic acid using $N, N^{\prime}$-dicyclohexylcarbodimide (DCC) and 1-hydroxybenzotriazole (HOBt) in methylene chloride to afford tertiary cyanoacetamides $\mathbf{5} \mathbf{a}$ and $\mathbf{5 b}$ in $46 \% \sim 63 \%$ yields.

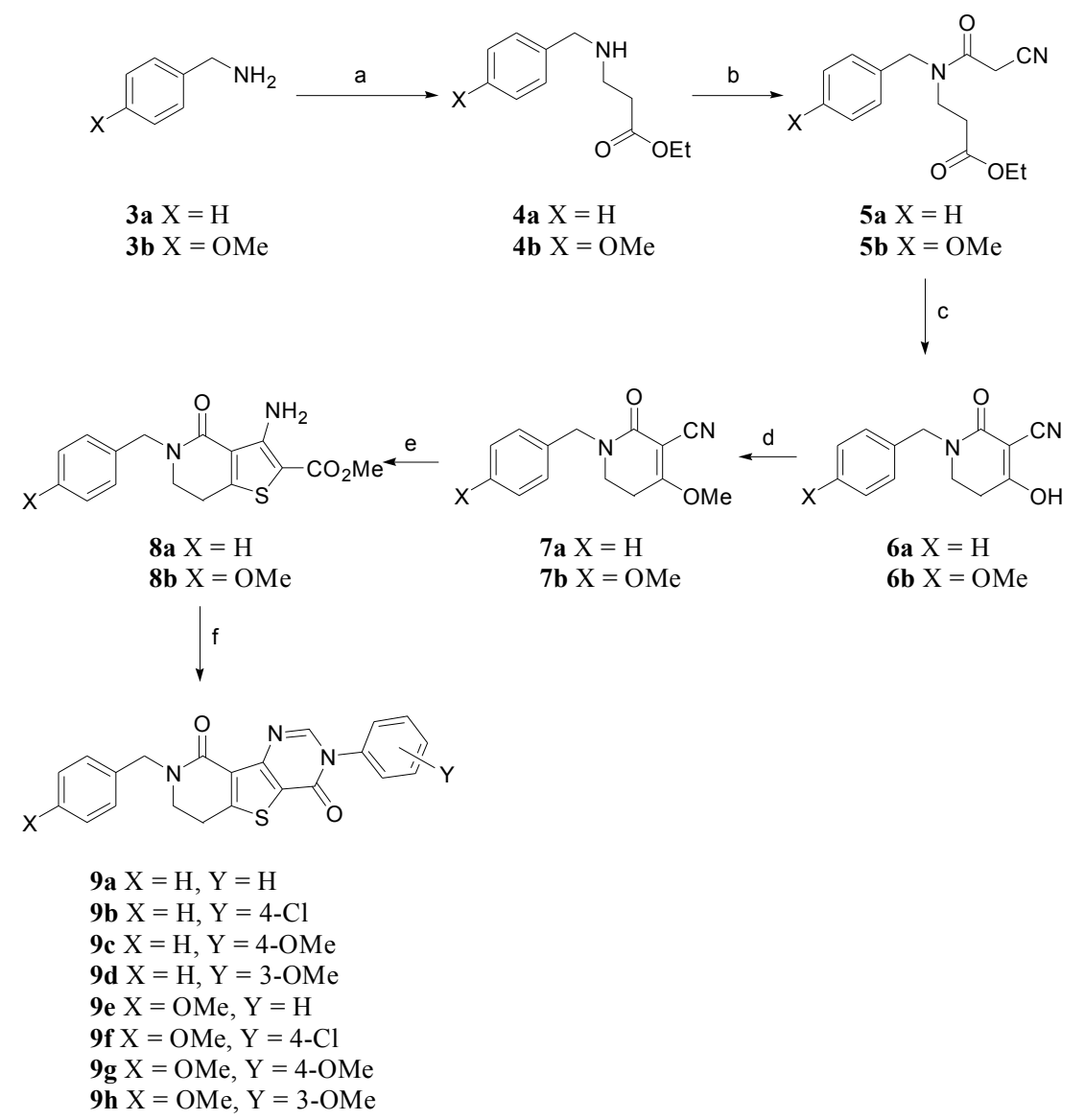

Reagents and conditions: (a) ethyl acrylate, $\mathrm{Cu}(\mathrm{OAc})_{2}, \mathrm{H}_{2} \mathrm{O}$, rt; (b) cyanoacetic acid, DCC, $\mathrm{HOBt}$, DCM, $0{ }^{\circ} \mathrm{C}$ to rt; (c) Amberlyst A-26 resin, $\mathrm{MeOH}$, rt; (d) $\mathrm{Me}_{2} \mathrm{SO}_{4}, \mathrm{NaH}, \mathrm{THF}$, rt to $40{ }^{\circ} \mathrm{C}$; (e) $\mathrm{HSCH}_{2} \mathrm{CO}_{2} \mathrm{Me}, \mathrm{NaOMe}$, $\mathrm{MeOH}, 65^{\circ} \mathrm{C}$; (f) anilines, $\mathrm{CH}(\mathrm{OEt})_{3}, \mathrm{AcOH}, 160^{\circ} \mathrm{C}$, sealed tube.

Scheme 1. Synthesis of $N^{3}$-aryl- $N^{8}$-benzyldihydropyridothienopyrimidin-4,9-diones $9 \mathbf{a}-\mathbf{9 h}$.

To form the dihydropyridone ring, the cyanoacetamides $\mathbf{5 a}$ and $\mathbf{5 b}$ underwent modified Dieckmann condensation under acidic conditions using Amberlyst A-26 resin as catalyst to give $\mathbf{6 a}$ and $\mathbf{6 b}$ in $55 \% \sim 64 \%$ yields [8]. The hydroxy groups of the compounds $\mathbf{6 a}$ and $\mathbf{6 b}$ were methylated by dimethyl sulfate and $\mathrm{NaH}$ (37\% 63\% yields). The resulting methylated compounds $7 \mathbf{a}$ and $7 \mathbf{b}$ were then subjected to a series of heterocyclization reactions $[9,10]$ to give the desired dihydropyridothienopyrimidin-4,9diones $9 \mathbf{a} \sim 9 \mathbf{h}$ in $20 \% \sim 67 \%$ combined yields.

The synthesized compounds $9 \mathbf{9} \sim 9 \mathbf{h}$ were biologically evaluated against Chem-3 cells with stably expressing mGluR1 which has been considered as a potential target for treating neuropathic pain [11], and the results are summarized in Table 1. Percent inhibitions (\%-inhibitions) for all the synthesized compounds were measured at $1 \mu \mathrm{M}$. Overall, the dihydropyridothienopyrimidin-4,9-dione derivatives showed low to moderate inhibitory activity compared with the reference compound (93.37\% inhibition) [9]: 
the compounds 9a, 9d, and 9f showed moderate inhibitory activity against mGluR1 with 23.04\%, $16.54 \%$, and $18.47 \%$ inhibition, respectively, while others showed negligible inhibitory activities.

Table 1. Inhibitory activities of the synthesized dihydropyridothienopyrimidin-4,9-diones 9 against mGluR1.

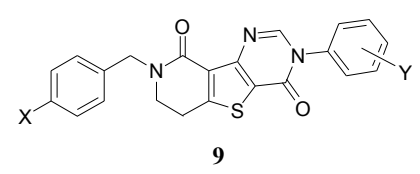

\begin{tabular}{|c|c|c|c|c|}
\hline Entry & Compound & $\mathbf{X}$ & $\mathbf{Y}$ & $\%$ Inhibition at $1 \mu M^{a}$ \\
\hline 1 & $9 a$ & $\mathrm{H}$ & $\mathrm{H}$ & $23.04 \%$ \\
\hline 2 & $9 b$ & $\mathrm{H}$ & $4-\mathrm{Cl}$ & $-1.59 \%$ \\
\hline 3 & $9 \mathrm{c}$ & $\mathrm{H}$ & 4-OMe & $3.15 \%$ \\
\hline 4 & 9d & $\mathrm{H}$ & 3-OMe & $16.54 \%$ \\
\hline 5 & $9 e$ & $\mathrm{OMe}$ & $\mathrm{H}$ & $8.29 \%$ \\
\hline 6 & 9f & $\mathrm{OMe}$ & $4-\mathrm{Cl}$ & $18.47 \%$ \\
\hline 7 & 9g & $\mathrm{OMe}$ & 4-OMe & $10.35 \%$ \\
\hline 8 & $9 \mathrm{~h}$ & $\mathrm{OMe}$ & 3-OMe & $11.86 \%$ \\
\hline 9 & \multicolumn{3}{|c|}{ Reference compound ${ }^{b}$} & $93.37 \%$ \\
\hline
\end{tabular}

a. \%-Inhibition of compounds at $1 \mu \mathrm{M}$ against mGluR $1 ;{ }^{b}$ : the reference compound is shown below.

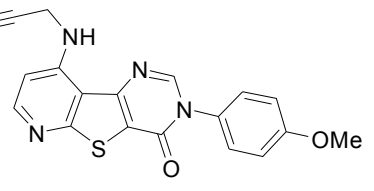

\section{Experimental Section}

\subsection{General Methods}

All reactions were carried out under dry nitrogen or argon unless otherwise indicated. Commercially available reagents were used without further purification. Solvents and gases were dried according to standard procedures. Organic solvents were evaporated with reduced pressure using a rotary evaporator. Analytical thin layer chromatography (TLC) was performed using glass plates precoated with silica gel $(0.25 \mathrm{~mm})$. TLC plates were visualized by exposure to UV light (UV), and then were visualized with a $\mathrm{KMnO}_{4}$ stain followed by brief heating on hot plate. Flash column chromatography was performed using silica gel 60 (230-400 mesh, Merck, Kenilworth, NJ, USA) with the indicated solvents. The melting points of solid products was measured by using capillary tubes for the determination of melting points (Marienfeld-Superior, Lauda-Königshofen, Germany) and an automated melting point system (OptiMelt, Stanford Research Systems, Inc. Sunnyvale, CA, USA). The tubes containing solid compounds were inserted in OptiMelt, and the temperature was slowly raised from $100{ }^{\circ} \mathrm{C}$ to $400{ }^{\circ} \mathrm{C}$. ${ }^{1} \mathrm{H}$ - and ${ }^{13} \mathrm{C}$-NMR spectra were recorded on Bruker 300 or 400 spectrometers (Bruker Co. Billerica, MA, USA). ${ }^{1} \mathrm{H}-\mathrm{NMR}$ spectra are reported as follows: chemical shift, multiplicity ( $\mathrm{s}=$ singlet, $\mathrm{d}=$ doublet, $\mathrm{t}=$ triplet, $\mathrm{q}=$ quartet, $\mathrm{m}=$ multiplet, $\mathrm{dd}=$ doublet of doublet, $\mathrm{td}=$ triplet of doublet, brs = broad singlet, brt $=$ broad triplet), integration, and coupling constant $(J)$ in Hertz $(\mathrm{Hz}) .{ }^{1} \mathrm{H}-\mathrm{NMR}$ chemical shifts are reported relative to $\mathrm{CDCl}_{3}(7.26 \mathrm{ppm}) .{ }^{13} \mathrm{C}-\mathrm{NMR}$ was recorded relative to the central line of $\mathrm{CDCl}_{3}$ 
(77.0 ppm). HRMS analyses were performed on Bruker compact $\mathrm{ESI}^{+}$positive mode. HPLC purifications were performed on an Alliance Waters $2489 \mathrm{UV} /$ Visible detector, e2695 Separations module using a Waters Xterra prep RP-18 $10 \mu \mathrm{M}, 10 \times 250 \mathrm{~mm}$ column (Waters Inc. Milford, MA, USA) and a $30 \%-100 \%$ acetonitrile in water solvent gradient.

Ethyl 3-(benzylamino)propanoate (4a). To a solution of benzylamine 3a (2.6 g, $24.0 \mathrm{mmol})$ in $\mathrm{H}_{2} \mathrm{O}$ $(100 \mathrm{~mL})$ were added ethyl acrylate $(2 \mathrm{~g}, 20.0 \mathrm{mmol})$, and $\mathrm{Cu}(\mathrm{OAc}) 2(182 \mathrm{mg}, 1.0 \mathrm{mmol})$. The resulting mixture was stirred at room temperature for $6 \mathrm{~h}$. After termination of the reaction, the solution was extracted with EtOAc, dried over $\mathrm{MgSO}_{4}$, and concentrated in vacuo. The residue was purified by silica gel column chromatography ( $n$-hexane-EtOAc $=1: 1$ ) to afford the desired product $4 \mathbf{a}(2.34 \mathrm{~g}$, $11.3 \mathrm{mmol}, 47 \%$ yield) as a yellow oil: ${ }^{1} \mathrm{H}-\mathrm{NMR}\left(300 \mathrm{MHz}, \mathrm{CDCl}_{3}\right) \delta 7.36-7.23(\mathrm{~m}, 5 \mathrm{H}), 4.14$ (q, $J=7.2 \mathrm{~Hz}, 2 \mathrm{H}), 3.81(\mathrm{~s}, 2 \mathrm{H}), 2.90(\mathrm{t}, J=6.3 \mathrm{~Hz}, 2 \mathrm{H}), 2.53(\mathrm{t}, J=6.3 \mathrm{~Hz}, 2 \mathrm{H}), 1.66(\mathrm{~s}, 1 \mathrm{H}), 1.25$ (t, $J=7.2 \mathrm{~Hz}, 3 \mathrm{H}) ;{ }^{13} \mathrm{C}-\mathrm{NMR}\left(100 \mathrm{MHz}, \mathrm{CDCl}_{3}\right) \delta 172.79,140.17,128.42,128.09,126.96,60.41,53.77$, 44.51, 34.79, 14.23; LC/MS (ESI ${ }^{+}$): $m / z$ : calcd for $\mathrm{C}_{12} \mathrm{H}_{17} \mathrm{NO}_{2}: 207.27,[\mathrm{M}+\mathrm{H}]^{+}$; found: 208.00 .

Ethyl 3-((4-methoxybenzyl)amino)propanoate (4b). Following the same procedure used for the synthesis of $\mathbf{4 a}$, the reaction of (4-methoxyphenyl)methanamine $\mathbf{3 b}(7.8 \mathrm{~mL}, 60.0 \mathrm{mmol})$, ethyl acrylate $(5 \mathrm{~g}$, $50.0 \mathrm{mmol}$ ), and $\mathrm{Cu}(\mathrm{OAc})_{2}(454 \mathrm{mg}, 2.5 \mathrm{mmol})$ gave the title compound $4 \mathbf{b}(6.72 \mathrm{~g}, 28.3 \mathrm{mmol}, 57 \%$ yield) as a brown oil: ${ }^{1} \mathrm{H}-\mathrm{NMR}\left(400 \mathrm{MHz}, \mathrm{CDCl}_{3}\right) \delta$ 7.24-7.22 (m, 2H), 6.87-6.85 (m, 2H), 4.13 (q, $J=7.1 \mathrm{~Hz}, 2 \mathrm{H}), 3.79(\mathrm{~s}, 3 \mathrm{H}), 3.74(\mathrm{~s}, 2 \mathrm{H}), 2.88(\mathrm{t}, J=6.4 \mathrm{~Hz}, 2 \mathrm{H}), 2.52(\mathrm{t}, J=6.4 \mathrm{~Hz}, 2 \mathrm{H}), 1.63(\mathrm{~s}, 1 \mathrm{H})$, $1.25(\mathrm{t}, J=7.2 \mathrm{~Hz}, 3 \mathrm{H}) ;{ }^{13} \mathrm{C}-\mathrm{NMR}\left(100 \mathrm{MHz}, \mathrm{CDCl}_{3}\right) \delta 172.80,158.66,132.30,129.26,113.81,60.40$, 55.26, 53.16, 44.41, 34.78, 14.22; LC/MS $\left(\mathrm{ESI}^{+}\right): m / z$ : calcd for $\mathrm{C}_{13} \mathrm{H}_{19} \mathrm{NO}_{3}: 237.30,[\mathrm{M}+\mathrm{H}]^{+}$; found: 238.30 .

Ethyl 3-(N-benzyl-2-cyanoacetamido)propanoate (5a). DCC (1.39 g, $6.75 \mathrm{mmol})$ were added to the a solution of ethyl 3-(benzylamino)propanoate 4a (1 g, $4.82 \mathrm{mmol}$ ), cyanoacetic acid (492 $\mathrm{mg}$, $5.79 \mathrm{mmol})$, and HOBt $(782 \mathrm{mg}, 5.79 \mathrm{mmol})$ in $\mathrm{MC}(30 \mathrm{~mL})$ at $0{ }^{\circ} \mathrm{C}$. The solution was stirred at room temperature for $12 \mathrm{~h}$. After termination of the reaction, the solution was filtered. The filtrate was washed with $\mathrm{NaHCO}_{3}$, and brine, dried over $\mathrm{MgSO}_{4}$, and concentrated in vacuo. The residue was purified by silica gel column chromatography ( $n$-hexane- EtOAc $=1: 2)$ to afford the desired product $\mathbf{5 a}(858 \mathrm{mg}$, $3.13 \mathrm{mmol}, 65 \%$ yield) as a yellow oil: ${ }^{1} \mathrm{H}$ NMR $\left(300 \mathrm{MHz}, \mathrm{CDCl}_{3}\right) \delta 7.42-7.15(\mathrm{~m}, 5 \mathrm{H}), 4.62(\mathrm{~d}$, $J=2.7 \mathrm{~Hz}, 2 \mathrm{H}), 4.12(\mathrm{q}, J=7.2 \mathrm{~Hz}, 2 \mathrm{H}), 3.87(\mathrm{~s}, 1 \mathrm{H}), 3.70(\mathrm{t}, J=6.6 \mathrm{~Hz}, 1 \mathrm{H}), 3.54(\mathrm{t}, J=6.3 \mathrm{~Hz}, 1 \mathrm{H})$, $3.47(\mathrm{~s}, 1 \mathrm{H}), 2.66(\mathrm{t}, J=6.6 \mathrm{~Hz}, 1 \mathrm{H}), 2.56(\mathrm{t}, J=6.6 \mathrm{~Hz}, 1 \mathrm{H}), 1.28-1.22(\mathrm{~m}, 3 \mathrm{H}) ;{ }^{13} \mathrm{C}-\mathrm{NMR}(100 \mathrm{MHz}$, $\mathrm{CDCl}_{3}$ ) (isomers) $\delta 171.71,171.07,162.63,162.57,136.24,135.39,129.34,128.86,128.23,127.95$, $127.85,126.08,114.42,113.92,61.27,60.81,52.94,48.35,44.11,43.00,32.55,32.51,25.35,25.23$, 14.16, 14.10; $\mathrm{LC} / \mathrm{MS}\left(\mathrm{ESI}^{+}\right): m / z$ : calcd for $\mathrm{C}_{15} \mathrm{H}_{18} \mathrm{~N}_{2} \mathrm{O}_{3}: 274.32,[\mathrm{M}+\mathrm{H}]^{+}$; found: 275.15 .

Ethyl 3-(2-cyano-N-(4-methoxybenzyl)acetamido)propanoate (5b). Following the same procedure used for the synthesis of 5a, the reaction of ethyl 3-((4-methoxybenzyl)amino)propanoate $\mathbf{4 b}$ (6.72 $\mathrm{g}$, $28.3 \mathrm{mmol})$, cyanoacetic acid $(2.89 \mathrm{~g}, 34.0 \mathrm{mmol})$, DCC $(8.17 \mathrm{~g}, 39.6 \mathrm{mmol})$ and HOBt $(4.59 \mathrm{~g}$, $34.0 \mathrm{mmol})$ in $\mathrm{MC}(125 \mathrm{~mL})$ gave the title compound $\mathbf{5 b}(3.99 \mathrm{~g}, 13.1 \mathrm{mmol}, 46 \%$ yield $)$ as a yellow oil: ${ }^{1} \mathrm{H}-\mathrm{NMR}\left(300 \mathrm{MHz}, \mathrm{CDCl}_{3}\right) \delta 7.13(\mathrm{dd}, J=8.6,26.1 \mathrm{~Hz}, 2 \mathrm{H}), 6.88(\mathrm{dd}, J=8.6,16.6 \mathrm{~Hz}, 2 \mathrm{H}), 4.55$ (s, 2H), 4.12 (q, $J=7.1 \mathrm{~Hz}, 2 \mathrm{H}), 3.83-3.80(\mathrm{~m}, 4 \mathrm{H}), 3.67(\mathrm{t}, J=6.6 \mathrm{~Hz}, 1 \mathrm{H}), 3.51(\mathrm{t}, J=6.4 \mathrm{~Hz}, 1 \mathrm{H}), 3.47$ 
$(\mathrm{s}, 1 \mathrm{H}), 2.64(\mathrm{t}, J=6.7 \mathrm{~Hz}, 1 \mathrm{H}), 2.56(\mathrm{t}, J=6.4 \mathrm{~Hz}, 1 \mathrm{H}), 1.28-1.22(\mathrm{~m}, 3 \mathrm{H}) ;{ }^{13} \mathrm{C}-\mathrm{NMR}(100 \mathrm{MHz}$, $\mathrm{CDCl}_{3}$ ) (isomers) $\delta 171.70,171.07,162.48,162.41,159.53,159.29,129.46,128.26,127.52,127.13$, 114.70, 114.43, 114.22, 113.99, 61.25, 60.79, 55.37, 55.29, 52.44, 47.70, 43.81, 42.69, 32.51, 25.36, 25.24, 14.15, 14.09; LC/MS (ESI $\left.{ }^{+}\right): m / z$ : calcd for $\mathrm{C}_{16} \mathrm{H}_{20} \mathrm{~N}_{2} \mathrm{O}_{4}: 304.35,[\mathrm{M}+\mathrm{H}]^{+}$; found: 305.15 .

Benzyl-4-hydroxy-2-oxo-1,2,5,6-tetrahydropyridine-3-carbonitrile (6a). To a solution of ethyl 3-( $N$-benzyl-2-cyanoacetamido)propanoate 5 a $(850 \mathrm{mg}, 3.10 \mathrm{mmol})$ in $\mathrm{MeOH}(20 \mathrm{~mL})$ were added Amberlyst A-26 resin (1.5 g). The resulting mixture was stirred at room temperature for $16 \mathrm{~h}$. After termination of the reaction, the resin was filtered and washed with $\mathrm{MeOH}$. Then, the resin was added to the solution of TFA $(2 \mathrm{~mL})$ in $\mathrm{MeOH}(20 \mathrm{~mL})$, and stirred at room temperature for $30 \mathrm{~min}$. The mixture solution was filtered, and concentrated in vacuo to afford the desired product $6 \mathbf{a}(390 \mathrm{mg}, 1.71 \mathrm{mmol}$, 55\% yield) as a white solid: mp $225-227{ }^{\circ} \mathrm{C} ;{ }^{1} \mathrm{H}-\mathrm{NMR}$ (300 MHz, DMSO-d6) $\delta 7.37-7.24(\mathrm{~m}, 5 \mathrm{H}), 4.51$ $(\mathrm{s}, 2 \mathrm{H}), 3.30(\mathrm{t}, J=7.2 \mathrm{~Hz}, 2 \mathrm{H}), 2.68(\mathrm{t}, J=6.9 \mathrm{~Hz}, 2 \mathrm{H}) ;{ }^{13} \mathrm{C}-\mathrm{NMR}\left(100 \mathrm{MHz}, \mathrm{DMSO}-d_{6}\right) \delta 181.05$, 163.16, 137.98, 128.97, 128.05 127.60, 115.57, 83.49, 49.35, 42.82, 29.01; LC/MS $\left(\mathrm{ESI}^{+}\right): \mathrm{m} / z$ : calcd for $\mathrm{C}_{13} \mathrm{H}_{12} \mathrm{~N}_{2} \mathrm{O}_{2}: 228.25,[\mathrm{M}+\mathrm{H}]^{+}$; found: 229.15 .

4-Hydroxy-1-(4-methoxybenzyl)-2-oxo-1,2,5,6-tetrahydropyridine-3-carbonitrile (6b). Following the same procedure used for the synthesis of $\mathbf{6 a}$, the reaction of ethyl 3-(2-cyano- $N$-(4methoxybenzyl)acetamido)propanoate $\mathbf{5 b}(200 \mathrm{mg}, 0.66 \mathrm{mmol})$, Amberlyst A-26 resin (354 mg), and TFA $(2 \mathrm{~mL})$ in $\mathrm{MeOH}(10 \times 2 \mathrm{~mL})$ gave the title compound $\mathbf{6 b}(108.7 \mathrm{mg}, 0.42 \mathrm{mmol}, 64 \%$ yield $)$ as a white solid: mp $170-173{ }^{\circ} \mathrm{C} ;{ }^{1} \mathrm{H}-\mathrm{NMR}$ (400 MHz, DMSO-d6) $\delta$ 7.30-7.26 (m, 2H), 7.01-6.98 (m, 2H), $4.53(\mathrm{~s}, 2 \mathrm{H}), 3.83(\mathrm{~s}, 3 \mathrm{H}), 3.36(\mathrm{t}, J=7.1 \mathrm{~Hz}, 2 \mathrm{H}), 2.74(\mathrm{t}, J=7.0 \mathrm{~Hz}, 2 \mathrm{H}) ;{ }^{13} \mathrm{C}-\mathrm{NMR}(100 \mathrm{MHz}$, DMSO-d6) $\delta 180.87,163.02,158.95,129.82,129.56,115.54,114.37,83.59,55.52,48.70,42.53,28.93$; LC/MS $\left(\mathrm{ESI}^{+}\right): m / z$ : calcd for $\mathrm{C}_{14} \mathrm{H}_{14} \mathrm{~N}_{2} \mathrm{O}_{3}: 258.28,[\mathrm{M}+\mathrm{H}]^{+}$; found: 259.15 .

1-Benzyl-4-methoxy-2-oxo-1,2,5,6-tetrahydropyridine-3-carbonitrile (7a). To a solution of benzyl-4hydroxy-2-oxo-1,2,5,6-tetrahydropyridine-3-carbonitrile 6a (100 mg, $0.44 \mathrm{mmol}$ ) and THF (3 mL), NaH ( $21 \mathrm{mg}, 0.88 \mathrm{mmol}$ ) was added and the reaction mixture was stirred at room temperature for $2 \mathrm{~h}$. Then dimethylsulfate $(0.071 \mathrm{~mL}, 0.75 \mathrm{mmol})$ was added to the mixture and the resulting solution was stirred at $40{ }^{\circ} \mathrm{C}$ for $12 \mathrm{~h}$. The mixture was quenched with $\mathrm{H}_{2} \mathrm{O}$ and evaporated. Then it was diluted with EtOAc, extracted with EtOAc, and dried over $\mathrm{MgSO}_{4}$ and concentrated, the crude product was purified by silica gel column chromatography ( $n$-hexane-EtOAc $=1: 1)$ to give product $7 \mathbf{a}(67 \mathrm{mg}, 0.28 \mathrm{mmol}, 63 \%$ yield) as a white solid: $\mathrm{mp} 153-157{ }^{\circ} \mathrm{C}$; ${ }^{1} \mathrm{H}-\mathrm{NMR}\left(300 \mathrm{MHz}, \mathrm{CDCl}_{3}\right) \delta$ 7.37-7.26 (m, 5H), $4.62(\mathrm{~s}, 2 \mathrm{H}), 4.14$ $(\mathrm{s}, 3 \mathrm{H}), 3.33(\mathrm{t}, J=6.9 \mathrm{~Hz}, 2 \mathrm{H}), 2.61(\mathrm{t}, J=6.9 \mathrm{~Hz}, 2 \mathrm{H}) ;{ }^{13} \mathrm{C}-\mathrm{NMR}\left(100 \mathrm{MHz}, \mathrm{CDCl}_{3}\right) \delta 177.55,162.08$, 136.66, 128.77, 128.19, 127.76, 114.15, 86.77, 58.13, 49.85, 42.07, 26.91; LC/MS (ESI $\left.{ }^{+}\right): m / z$ : calcd for $\mathrm{C}_{14} \mathrm{H}_{14} \mathrm{~N}_{2} \mathrm{O}_{2}: 242.28,[\mathrm{M}+\mathrm{H}]^{+}$; found: 243.15.

4-Methoxy-1-(4-methoxybenzyl)-2-oxo-1,2,5,6-tetrahydropyridine-3-carbonitrile (7b). Following the same procedure used for the synthesis of 7a, the reaction of 4-hydroxy-1-(4-methoxybenzyl)-2-oxo1,2,5,6-tetrahydropyridine-3-carbonitrile $6 \mathbf{b}(1.74 \mathrm{~g}, 6.70 \mathrm{mmol}), \mathrm{NaH}$ (321 $\mathrm{mg}, 13.4 \mathrm{mmol})$, and dimethylsulfate $(1.1 \mathrm{ml}, 11.4 \mathrm{mmol})$ gave the title compound $7 \mathbf{b}(677 \mathrm{mg}, 2.45 \mathrm{mmol}, 37 \%$ yield $)$ as a white solid: $\mathrm{mp} 145-148{ }^{\circ} \mathrm{C} ;{ }^{1} \mathrm{H}-\mathrm{NMR}\left(300 \mathrm{MHz}, \mathrm{CDCl}_{3}\right) \delta 7.23(\mathrm{~d}, J=8.1 \mathrm{~Hz}, 2 \mathrm{H}), 6.88(\mathrm{~d}, J=8.4 \mathrm{~Hz}$, 2H), $4.57(\mathrm{~s}, 2 \mathrm{H}), 4.14(\mathrm{~s}, 3 \mathrm{H}), 3.82(\mathrm{~s}, 3 \mathrm{H}), 3.35(\mathrm{t}, J=6.7 \mathrm{~Hz}, 2 \mathrm{H}), 2.65(\mathrm{t}, J=6.7 \mathrm{~Hz}, 2 \mathrm{H}) ;{ }^{13} \mathrm{C}-\mathrm{NMR}$ 
(75 MHz, $\left.\mathrm{CDCl}_{3}\right) \delta 177.20,161.95,159.28,129.62,128.73,114.16,86.85,58.14,55.31,49.29,41.87$, 27.10; LC/MS (ESI ${ }^{+}$): $m / z$ : calcd for $\mathrm{C}_{15} \mathrm{H}_{16} \mathrm{~N}_{2} \mathrm{O}_{3}: 272.30,[\mathrm{M}+\mathrm{H}]^{+}$; found: 273.15 .

Methyl 3-amino-5-benzyl-4-oxo-4,5,6,7-tetrahydrothieno[3,2-c]pyridine-2-carboxylate (8a). NaOMe $(5 \mathrm{M}$ in $\mathrm{MeOH}, 36.0 \mathrm{~mL}, 17.9 \mathrm{mmol})$ in $\mathrm{MeOH}$ followed by methyl thioglycolate $(1.8 \mathrm{~mL}, 20.5 \mathrm{mmol})$ were added to 1-benzyl-4-methoxy-2-oxo-1,2,5,6-tetrahydropyridine-3-carbonitrile 7a (3.1 g, $12.8 \mathrm{mmol})$. The reaction mixture was stirred and refluxed at $65{ }^{\circ} \mathrm{C}$ for $12 \mathrm{~h}$. After the reaction, the mixture was filtered using Celite and washed with MC. The crude compound was then purified by by silica gel column chromatography $(n$-hexane-EtOAc $=4: 1)$ to give white solid product 8a $(2.44 \mathrm{~g}, 7.38 \mathrm{mmol}$, $58 \%$ yield) as a yellowish solid: $\mathrm{mp} 110-112{ }^{\circ} \mathrm{C} ;{ }^{1} \mathrm{H}-\mathrm{NMR}\left(300 \mathrm{MHz}, \mathrm{CDCl}_{3}\right) \delta 7.37-7.27(\mathrm{~m}, 5 \mathrm{H})$, 6.91 (brs, 2H), 4.70 (s, 2H), $3.82(\mathrm{~s}, 3 \mathrm{H}), 3.53(\mathrm{t}, J=6.9 \mathrm{~Hz}, 2 \mathrm{H}), 2.93(\mathrm{t}, J=6.9 \mathrm{~Hz}, 2 \mathrm{H}) ;{ }^{13} \mathrm{C}-\mathrm{NMR}$ $\left(100 \mathrm{MHz}, \mathrm{CDCl}_{3}\right) \delta 164.55,162.73,153.78,151.00,137.12,128.77,127.99,127.63,119.74,96.83$, 51.16, 49.03, 45.82, 25.00; LC/MS (ESI $\left.{ }^{+}\right): m / z$ : calcd for $\mathrm{C}_{16} \mathrm{H}_{16} \mathrm{~N}_{2} \mathrm{O} 3 \mathrm{~S}: 316.38,[\mathrm{M}+\mathrm{H}]^{+}$; found: 317.05 .

Methyl 3-amino-5-(4-methoxybenzyl)-4-oxo-4,5,6,7-tetrahydrothieno[3,2-c]pyridine-2-carboxylate (8b). Following the same procedure used for the synthesis of $\mathbf{8 a}$, the reaction of 4-methoxy-1-(4methoxybenzyl)-2-oxo-1,2,5,6-tetrahydropyridine-3-carbonitrile $7 \mathbf{b}$ (354 mg, $1.30 \mathrm{mmol}), \mathrm{NaOMe}(5 \mathrm{M}$ in $\mathrm{MeOH}, 0.40 \mathrm{~mL}, 2.08 \mathrm{mmol})$, and methyl thioglycolate $(0.21 \mathrm{~mL}, 2.34 \mathrm{mmol})$ gave the title compound 8b (281 mg, $0.81 \mathrm{mmol}, 62 \%$ yield) as a yellow oil: ${ }^{1} \mathrm{H}-\mathrm{NMR}\left(400 \mathrm{MHz}, \mathrm{CDCl}_{3}\right) \delta 7.24-7.22(\mathrm{~m}, 2 \mathrm{H})$, 6.88-6.86 (m, 2H), $4.62(\mathrm{~s}, 2 \mathrm{H}), 3.82(\mathrm{~s}, 3 \mathrm{H}), 3.80(\mathrm{~s}, 3 \mathrm{H}), 3.50(\mathrm{t}, J=6.9 \mathrm{~Hz}, 2 \mathrm{H}), 2.91(\mathrm{t}, J=6.9 \mathrm{~Hz}$, $2 \mathrm{H}) ;{ }^{13} \mathrm{C}-\mathrm{NMR}\left(100 \mathrm{MHz}, \mathrm{CDCl}_{3}\right) \delta 164.52,162.63,159.13,153.74,150.99,129.37,129.13,119.77$, 114.11, 96.80, 55.28, 51.12, 48.38, 45.59, 24.95; LC/MS (ESI $\left.{ }^{+}\right): m / z$ : calcd for $\mathrm{C}_{17} \mathrm{H}_{18} \mathrm{~N}_{2} \mathrm{O}_{4} \mathrm{~S}: 346.40$, $[\mathrm{M}+\mathrm{H}]^{+}$; found: 347.05 .

8-Benzyl-3-phenyl-7,8-dihydropyrido[3',4':4,5]thieno[3,2-d]pyrimidine-4,9(3H,6H)-dione (9a). To a pressure bottle containing methyl 3-amino-5-benzyl-4-oxo-4,5,6,7-tetrahydrothieno[3,2-c]pyridine-2carboxylate 8a $(1 \mathrm{~g}, 3.03 \mathrm{mmol}), \mathrm{CH}(\mathrm{OEt})_{3}(10 \mathrm{~mL})$ was added, followed by aniline $(0.524 \mathrm{~mL}$, $5.75 \mathrm{mmol})$ and $\mathrm{AcOH}(1 \mathrm{~mL})$. The reaction mixture was stirred and refluxed at $160{ }^{\circ} \mathrm{C}$ for $18 \mathrm{~h}$. After the reaction, the mixture was evaporated then solidified with EtOAc and $\mathrm{Et}_{2} \mathrm{O}$. The produced solid was filtered and dried in vacuo to give the title product $9 \mathrm{a}$ (743 $\mathrm{mg}, 1.92 \mathrm{mmol}, 63 \%$ yield) as a reddish solid: mp 172-173 ${ }^{\circ} \mathrm{C} ;{ }^{1} \mathrm{H}-\mathrm{NMR}\left(300 \mathrm{MHz}, \mathrm{DMSO}-d_{6}\right) \delta 8.44-7.51(\mathrm{~m}, 5 \mathrm{H}), 7.39-7.26(\mathrm{~m}, 5 \mathrm{H}), 4.71(\mathrm{~s}, 2 \mathrm{H})$, $3.62(\mathrm{t}, J=6.6 \mathrm{~Hz}, 2 \mathrm{H}), 3.23(\mathrm{t}, J=6.6 \mathrm{~Hz}, 2 \mathrm{H}) ;{ }^{13} \mathrm{C}-\mathrm{NMR}\left(100 \mathrm{MHz}, \mathrm{DMSO}-d_{6}\right) \delta 159.61,156.61$, $156.48,154.82,149.69,138.26,137.32,129.71,129.49,129.20,128.08,128.02,127.63,126.01,122.13$, 49.19, 46.26, 25.79; HRMS (ESI TOF-mass) calcd for $\mathrm{C}_{22} \mathrm{H}_{17} \mathrm{~N}_{3} \mathrm{O}_{2} \mathrm{~S}[\mathrm{M}+\mathrm{Na}]^{+}$410.0934, found 410.0936; purity (HPLC) $83.91 \%, t_{\mathrm{R}} 18.00 \mathrm{~min}$.

8-Benzyl-3-(4-chlorophenyl)-7,8-dihydropyrido[3',4':4,5]thieno[3,2-d]pyrimidine-4,9(3H,6H)-dione (9b). Following the same procedure used for the synthesis of $9 \mathbf{a}$, the reaction of methyl 3-amino-5-benzyl-4oxo-4,5,6,7-tetrahydrothieno[3,2-c]pyridine-2-carboxylate $\mathbf{8 a}(100 \mathrm{mg}, 0.32 \mathrm{mmol}), \mathrm{CH}(\mathrm{OEt})_{3}(1 \mathrm{~mL})$, 4-chloroaniline $(72.7 \mathrm{mg}, 0.57 \mathrm{mmol})$ and $\mathrm{AcOH}(0.1 \mathrm{~mL})$ gave the title compound $\mathbf{9 b}(89 \mathrm{mg}$, $0.21 \mathrm{mmol}, 66 \%$ yield) as a yellowish solid: mp 220-223 ${ }^{\circ} \mathrm{C} ;{ }^{1} \mathrm{H}-\mathrm{NMR}\left(400 \mathrm{MHz}, \mathrm{CDCl}_{3}\right) \delta 8.27$ (s, 1H), 7.54-7.27 (m, 9H), $4.80(\mathrm{~s}, 2 \mathrm{H}), 3.63(\mathrm{t}, J=6.7 \mathrm{~Hz}, 2 \mathrm{H}), 3.13(\mathrm{t}, J=6.8 \mathrm{~Hz}, 2 \mathrm{H}) ;{ }^{13} \mathrm{C}-\mathrm{NMR}$ $\left(100 \mathrm{MHz} \mathrm{CDCl}_{3}\right) \delta 160.06,156.36,155.24,154.74,148.31,137.33,135.55,135.10,129.93,128.72$, 
128.46, 128.39, 127.65, 126.17, 122.65, 49.53, 45.64, 25.89; HRMS (ESI TOF-mass) calcd for $\mathrm{C}_{22} \mathrm{H}_{16} \mathrm{ClN}_{3} \mathrm{O}_{2} \mathrm{~S}[\mathrm{M}+\mathrm{Na}]^{+} 444.0544$, found 444.0545; purity (HPLC) $100.00 \%$, $t \mathrm{R} 19.21 \mathrm{~min}$.

8-Benzyl-3-(4-methoxyphenyl)-7,8-dihydropyrido [3',4':4,5] thieno[3,2-d]pyrimidine-4,9(3H,6H)-dione(9c). Following the same procedure used for the synthesis of 9a, the reaction of methyl 3-amino-5-benzyl-4oxo-4,5,6,7-tetrahydrothieno[3,2-c]pyridine-2-carboxylate $\mathbf{8 a}(100 \mathrm{mg}, 0.32 \mathrm{mmol}), \mathrm{CH}(\mathrm{OEt})_{3}(1 \mathrm{~mL})$, p-anisidine $(72.7 \mathrm{mg}, 0.57 \mathrm{mmol})$ and $\mathrm{AcOH}(0.1 \mathrm{~mL})$ gave the title compound $9 \mathrm{c}(44 \mathrm{mg}, 0.11 \mathrm{mmol}$, $33 \%$ yield) as a reddish solid: $\mathrm{mp} 223-225{ }^{\circ} \mathrm{C} ;{ }^{1} \mathrm{H}-\mathrm{NMR}\left(400 \mathrm{MHz}, \mathrm{CDCl}_{3}\right) \delta 8.27(\mathrm{~s}, 1 \mathrm{H}), 7.40-7.04$ $(\mathrm{m}, 9 \mathrm{H}), 4.81(\mathrm{~s}, 2 \mathrm{H}), 3.87(\mathrm{~s}, 3 \mathrm{H}), 3.63(\mathrm{t}, J=6.7 \mathrm{~Hz}, 2 \mathrm{H}), 3.14(\mathrm{t}, J=6.7 \mathrm{~Hz}, 2 \mathrm{H}) ;{ }^{13} \mathrm{C}-\mathrm{NMR}$ $\left(100 \mathrm{MHz}, \mathrm{CDCl}_{3}\right) \delta 160.19,160.17,156.84,154.92,154.81,149.05,137.41,129.35,128.71,128.39$, 128.23, 127.61, 126.13, 122.79, 114.88, 55.64, 49.48, 45.64, 25.89; HRMS (ESI TOF-mass) calcd for $\mathrm{C}_{23} \mathrm{H}_{19} \mathrm{~N}_{3} \mathrm{O}_{3} \mathrm{~S}[\mathrm{M}+\mathrm{Na}]^{+} 440.1039$, found 440.1039; purity (HPLC) $100.00 \%$, $t \mathrm{R} 18.09 \mathrm{~min}$.

8-Benzyl-3-(3-methoxyphenyl)-7,8-dihydropyrido[3',4':4,5] thieno[3,2-d]pyrimidine-4,9(3H,6H)-dione (9d). Following the same procedure used for the synthesis of 9a, the reaction of methyl 3-amino-5benzyl-4-oxo-4,5,6,7-tetrahydrothieno[3,2-c]pyridine-2-carboxylate 8a (200 mg, $0.63 \mathrm{mmol}), \mathrm{CH}(\mathrm{OEt})_{3}$ $(2 \mathrm{~mL}), m$-anisidine $(135 \mu \mathrm{L}, 1.21 \mathrm{mmol})$ and $\mathrm{AcOH}(0.2 \mathrm{~mL})$ gave the title compound $9 \mathrm{~d}(53 \mathrm{mg}, 0.13$ mmol, 20\% yield) as a white solid: $\mathrm{mp} 223-226{ }^{\circ} \mathrm{C} ;{ }^{1} \mathrm{H}-\mathrm{NMR}\left(400 \mathrm{MHz}, \mathrm{CDCl}_{3}\right) \delta 8.27(\mathrm{~s}, 1 \mathrm{H})$, 7.47-6.95 (m, 9H), $4.81(\mathrm{~s}, 2 \mathrm{H}), 3.85(\mathrm{~s}, 3 \mathrm{H}), 3.63(\mathrm{t}, J=6.8 \mathrm{~Hz}, 2 \mathrm{H}), 3.14(\mathrm{t}, J=6.7 \mathrm{~Hz}, 2 \mathrm{H}) ;{ }^{13} \mathrm{C}-\mathrm{NMR}$ $\left(100 \mathrm{MHz}, \mathrm{CDCl}_{3}\right) \delta 160.43,160.17,156.50,154.98,154.78,148.72,137.72,137.38,130.45,128.72$, $128.39,127.63,126.18,122.86,119.15,115.39,112.93,55.60,49.49,45.63,25.91$; HRMS (ESI TOF-mass) calcd for $\mathrm{C}_{23} \mathrm{H}_{19} \mathrm{~N}_{3} \mathrm{O}_{3} \mathrm{~S}[\mathrm{M}+\mathrm{Na}]^{+} 440.1039$, found 440.1038; purity (HPLC) $99.64 \%, t_{\mathrm{R}}$ $18.31 \mathrm{~min}$.

8-(4-Methoxybenzyl)-3-phenyl-7,8-dihydropyrido [3',4':4,5] thieno[3,2-d]pyrimidine-4,9(3H,6H)-dione (9e). Following the same procedure used for the synthesis of 9a, the reaction of methyl 3-amino-5-(4methoxybenzyl)-4-oxo-4,5,6,7-tetrahydrothieno[3,2-c]pyridine-2-carboxylate $\mathbf{8 b}$ (118 mg, $0.34 \mathrm{mmol}$ ), $\mathrm{CH}(\mathrm{OEt})_{3}(1.2 \mathrm{~mL})$, aniline $(62 \mu \mathrm{L}, 0.68 \mathrm{mmol})$ and $\mathrm{AcOH}(0.12 \mathrm{~mL})$ gave the title compound 9e (95 mg, $0.23 \mathrm{mmol}, 67 \%$ yield) as a yellowish solid: mp 227-230 ${ }^{\circ} \mathrm{C}$; ${ }^{1} \mathrm{H}-\mathrm{NMR}$ (400 MHz, DMSO-d6) $\delta 8.27(\mathrm{~s}, 1 \mathrm{H}), 7.58-6.85(\mathrm{~m}, 9 \mathrm{H}), 4.73(\mathrm{~s}, 2 \mathrm{H}), 3.79(\mathrm{~s}, 3 \mathrm{H}), 3.61(\mathrm{t}, J=6.7 \mathrm{~Hz}, 2 \mathrm{H}), 3.12(\mathrm{t}, J=6.8 \mathrm{~Hz}$, $2 \mathrm{H}) ;{ }^{13} \mathrm{C}-\mathrm{NMR}\left(100 \mathrm{MHz}, \mathrm{CDCl}_{3}\right) \delta 160.16,159.18,156.56,154.96,154.78,148.80,136.70,134.10$, 129.76, 129.70, 129.43, 127.06, 126.22, 122.87, 114.09, 55.31, 48.87, 45.43, 25.91; HRMS (ESI TOF-mass) calcd for $\mathrm{C}_{23} \mathrm{H}_{19} \mathrm{~N}_{3} \mathrm{O}_{3} \mathrm{~S}[\mathrm{M}+\mathrm{Na}]^{+}$440.1039, found 440.1039; purity (HPLC) $98.75 \%$, $t_{\mathrm{R}} 17.88 \mathrm{~min}$.

3-(4-Chlorophenyl)-8-(4-methoxybenzyl)-7,8-dihydropyrido[3',4':4,5] thieno[3,2-d]pyrimidine-4,9(3H,6H)dione (9f). Following the same procedure used for the synthesis of 9a, the reaction of methyl 3-amino5-(4-methoxybenzyl)-4-oxo-4,5,6,7-tetrahydrothieno[3,2-c]pyridine-2-carboxylate $\mathbf{8 b}$ (100 mg, $0.29 \mathrm{mmol}$ ), $\mathrm{CH}(\mathrm{OEt})_{3}(1 \mathrm{~mL})$, 4-chloroaniline $(74 \mathrm{mg}, 0.58 \mathrm{mmol})$ and $\mathrm{AcOH}(0.1 \mathrm{~mL})$ gave the title compound $9 \mathrm{f}$ (68 mg, $0.15 \mathrm{mmol}, 52 \%$ yield) as a yellowish solid: $\mathrm{mp} 269-272{ }^{\circ} \mathrm{C} ;{ }^{1} \mathrm{H}-\mathrm{NMR}\left(400 \mathrm{MHz}, \mathrm{CDCl}_{3}\right) \delta$ $8.24(\mathrm{~s}, 1 \mathrm{H}), 7.55-6.86(\mathrm{~m}, 8 \mathrm{H}), 4.74(\mathrm{~s}, 2 \mathrm{H}), 3.80(\mathrm{~s}, 3 \mathrm{H}), 3.62(\mathrm{t}, J=6.7 \mathrm{~Hz}, 2 \mathrm{H}), 3.13(\mathrm{t}, J=6.7 \mathrm{~Hz}$, $2 \mathrm{H}) ;{ }^{13} \mathrm{C}-\mathrm{NMR}\left(100 \mathrm{MHz}, \mathrm{CDCl}_{3}\right) \delta 159.98,159.17,156.36,155.19,154.72,148.27,135.53,135.11$, 
129.91, 129.77, 129.42, 128.47, 126.24, 122.61, 114.08, 55.31, 48.92, 45.46, 25.88; HRMS (ESI TOF-mass) calcd for $\mathrm{C}_{23} \mathrm{H}_{18} \mathrm{ClN}_{3} \mathrm{O}_{3} \mathrm{~S}[\mathrm{M}+\mathrm{Na}]^{+}$474.0650, found 474.0652; purity (HPLC) $100.00 \%, t \mathrm{R} 19.03 \mathrm{~min}$.

8-(4-Methoxybenzyl)-3-(4-methoxyphenyl)-7,8-dihydropyrido[3',4':4,5] thieno[3,2-d]pyrimidine$4,9(3 H, 6 H)$-dione $(\mathbf{9 g})$. Following the same procedure used for the synthesis of $\mathbf{9 a}$, the reaction of methyl 3-amino-5-(4-methoxybenzyl)-4-oxo-4,5,6,7-tetrahydrothieno[3,2-c]pyridine-2-carboxylate $\mathbf{8 b}$ (100 mg, $0.29 \mathrm{mmol}), \mathrm{CH}(\mathrm{OEt})_{3}(1 \mathrm{~mL}), \mathrm{p}$-anisidine $(73 \mathrm{mg}, 0.58 \mathrm{mmol})$ and $\mathrm{AcOH}(0.1 \mathrm{~mL})$ gave the title compound $9 \mathrm{~g}$ (67 mg, $0.15 \mathrm{mmol}, 51 \%$ yield) as a reddish solid: $\mathrm{mp} 225-228{ }^{\circ} \mathrm{C}$; ${ }^{1} \mathrm{H}-\mathrm{NMR}(400 \mathrm{MHz}$, $\left.\mathrm{CDCl}_{3}\right) \delta 8.25(\mathrm{~s}, 1 \mathrm{H}), 7.33-7.30(\mathrm{~m}, 4 \mathrm{H}), 7.06-6.84(\mathrm{~m}, 4 \mathrm{H}), 4.73(\mathrm{~s}, 2 \mathrm{H}), 3.86(\mathrm{~s}, 3 \mathrm{H}), 3.78(\mathrm{~s}, 3 \mathrm{H}), 3.60$ $(\mathrm{t}, J=6.7 \mathrm{~Hz}, 2 \mathrm{H}), 3.12(\mathrm{t}, J=6.7 \mathrm{~Hz}, 2 \mathrm{H}) ;{ }^{13} \mathrm{C}-\mathrm{NMR}\left(100 \mathrm{MHz}, \mathrm{CDCl}_{3}\right) \delta 160.18,160.16,159.17$, $156.85,154.83,154.82,149.04,129.76,129.47,129.35,128.20,126.23,122.82,114.89,114.08,55.63$, 55.31, 48.86, 45.44, 25.91; HRMS (ESI TOF-mass) calcd for $\mathrm{C}_{24} \mathrm{H}_{21} \mathrm{~N}_{3} \mathrm{O}_{4} \mathrm{~S}[\mathrm{M}+\mathrm{Na}]^{+} 470.1145$, found 470.1146; purity (HPLC) $100.00 \%, t_{\mathrm{R}} 17.95 \mathrm{~min}$.

\section{8-(4-Methoxybenzyl)-3-(3-methoxyphenyl)-7,8-dihydropyrido[3',4':4,5] thieno[3,2-d]pyrimidine-}

$4,9(3 H, 6 H)$-dione $(\mathbf{9 h})$. Following the same procedure used for the synthesis of $\mathbf{9 a}$, the reaction of methyl 3-amino-5-(4-methoxybenzyl)-4-oxo-4,5,6,7-tetrahydrothieno[3,2-c]pyridine-2-carboxylate $\mathbf{8 b}$ (100 mg, $0.29 \mathrm{mmol}), \mathrm{CH}(\mathrm{OEt})_{3}(1 \mathrm{~mL}), \mathrm{m}$-anisidine $(65 \mu \mathrm{L}, 0.58 \mathrm{mmol})$ and $\mathrm{AcOH}(0.1 \mathrm{~mL})$ gave the title compound $9 \mathrm{~h}$ (66 mg, $0.15 \mathrm{mmol}, 51 \%$ yield) as a yellowish solid: mp $171-175{ }^{\circ} \mathrm{C} ;{ }^{1} \mathrm{H}-\mathrm{NMR}(400 \mathrm{MHz}$, $\left.\mathrm{CDCl}_{3}\right) \delta 8.28(\mathrm{~s}, 1 \mathrm{H}), 7.46-6.84(\mathrm{~m}, 8 \mathrm{H}), 4.74(\mathrm{~s}, 2 \mathrm{H}), 3.85(\mathrm{~s}, 3 \mathrm{H}), 3.79(\mathrm{~s}, 3 \mathrm{H}), 3.61(\mathrm{t}, J=6.7 \mathrm{~Hz}$, 2H), $3.12(\mathrm{t}, J=6.8 \mathrm{~Hz}, 2 \mathrm{H}) ;{ }^{13} \mathrm{C}-\mathrm{NMR}\left(100 \mathrm{MHz}, \mathrm{CDCl}_{3}\right) \delta 160.42,160.12,159.16,156.51,154.98$, 154.72, 148.77, 137.71, 130.44, 129.76, 129.44, 126.20, 122.81, 119.15, 115.41, 114.08, 112.92, 55.60, 55.31, 48.87, 45.44, 25.89; HRMS (ESI TOF-mass) calcd for $\mathrm{C}_{24} \mathrm{H}_{21} \mathrm{~N}_{3} \mathrm{O}_{4} \mathrm{~S}[\mathrm{M}+\mathrm{Na}]^{+} 470.1145$, found 470.1146; purity (HPLC) $98.23 \%, t_{\mathrm{R}} 18.13 \mathrm{~min}$.

\section{2. mGluR1 Assay using FDSS6000}

Chem-3 cells which stably express mGluR1 were purchased from Milipore Co. (Billerica, MA, USA). Cells were grown in DMEM medium supplemented with $10 \%(\mathrm{v} / \mathrm{v})$ fetal bovine serum, penicillin $(100 \mathrm{U} / \mathrm{mL})$, streptomycin $(100 \mu \mathrm{g} / \mathrm{mL})$, and puromycin $(10 \mu \mathrm{g} / \mathrm{mL})$ at $37{ }^{\circ} \mathrm{C}$ in a humid atmosphere of $5 \% \mathrm{CO}_{2}$ and $95 \%$ air. For calcium assay, cells were harvested and dispensed into 96-well black wall clear bottom plates at a density of 40,000 cells per a well. After $18 \mathrm{~h}$ of incubation, cells were treated with Calcium-5 assay reagent, which is prepared by manufacture's instruction (Molecular Devices Co. Sunnyvale, CA, USA). During fluorescence-based FDSS6000 assay, mGluR1 was activated using a high concentration of L-glutamate $(10 \mu \mathrm{M})$ in HBSS, and proper concentrations of synthesized compounds were treated to cells $75 \mathrm{~s}$ before mGluR activation. All data were collected and analyzed using FDSS6000 and related software (Hamamatsu Photonics, Hamamatsu, Japan).

\section{Conclusions}

In this proof-of-concept study, a synthetic protocol was devised to allow access to a fused tricyclic heterocycle composed of dihydropyridone, thiophene and pyrimidinone. Also, even though only a small set of the dihydropyridothienopyrimidin-4,9-dione derivatives were synthesized, promising biological 
activity was identified in the title compounds. Thus, by using variously substituted benzylamines and anilines, an extensive structure-activity relationship study is warranted to investigate the pharmacological versatility of the dihydropyridothienopyrimidin-4,9-dione derivatives.

\section{Supplementary Materials}

Supplementary materials can be accessed at: http://www.mdpi.com/1420-3049/20/03/5074/s1.

\section{Acknowledgments}

This research was supported by the Basic Science Research Program (NRF-2013R1A1A2A10009907) funded by the National Research Foundation of Korea (NRF). Additional funding was provided by the Korea Institute of Science and Technology (KIST) Institutional Program (2E24510, 2E25473, and 2E25240).

\section{Author Contributions}

YK, JT, D-JB, and HC designed research; YK and MK performed research; YK, MK, MP, and KDP analyzed the data; YK and $\mathrm{HC}$ wrote the paper. All authors read and approved the final manuscript.

\section{Conflicts of Interest}

The authors declare no conflict of interest.

\section{References}

1. Wood, E.R.; Shewchuk, L.M.; Ellis, B.; Brignola, P.; Brashear, R.L.; Caferro, T.R.; Dickerson, S.H.; Dickson, H.D.; Donaldson, K.H.; Gaul, M.; et al. 6-Ethynylthieno[3,2-d]- and 6-ethynylthieno[2,3e]pyrimidin-4-anilines as tunable covalent modifiers of ErbB kinases. Proc. Natl. Acad. Sci. USA 2008, 105, 2773-2778.

2. Perspicace, E.; Jouan-Hureaux, V.; Ragno, R.; Ballante, F.; Sartini, S.; la Motta, C.; da Settimo, F.; Chen, B.; Kersch, G.; Schneider, S.; et al. Design, synthesis and biological evaluation of new classes of thieno[3,2- $d]$ pyrimidinone and thieno[1,2,3]triazine as inhibitor of vascular endothelial growth factor receptor-s (VEGFR-2). Eur. J. Med. Chem. 2013, 63, 765-781.

3. CAPRIE Streering Committee. A randomized, blinded, trial of clopidogrel versus aspirin in patients at risk of ischaemic events (CAPREI). Lancet 1996, 348, 1329-1339.

4. Sabatine, M.S. Clopidogrel in ST-elevation myocardial infarction. Eur. Heart J. 2006, 8, G31-G34.

5. Aalla, S.; Gilla, G.; Metil, D.S.; Anumula, R.R.; Vummenthala, P.R.; Padi, P.R. Process improvements of prasugrel hydrochloride: An adenosine diphosphate receptor antagonist. Org. Process Res. Dev. 2012, 16, 240-243.

6. Aradi, D.; Rideg, O.; Vorobcsuk, A.; Magyarlaki, T.; Magyari, B.; Konyi, A.; Pinter, T.; Horvath, I.G.; Komocsi, A. Justification of $150 \mathrm{mg}$ clopidogrel in patients with high on-clopidogrel platelet reactivity. Eur. J. Clin. Investig. 2012, 42, 384-392. 
7. Abdel-Wadood, F.K.; Abdel-Monem, M.I.; Fahmy, A.M.; Geies, A.A. Synthesis, reactions, and biological activities of some new thieno[3,2-c]quinoline and pyrrolo[3,2-c]quinoline derivatives. Arch. Pharm. Chem. Life Sci. 2014, 347, 142-152.

8. Kulkarni, B.A.; Ganesan, A. Ion-exchange resins for combinatorial synthesis: 2,4-pyrrolidinediones by Dieckmann condensation. Angew. Chem. Int. Ed. Engl. 1997, 36, 2454-2455.

9. Sasikumar, T.K.; Qiang, L.; Burnett, D.A.; Greenlee, W.J.; Li, C.; Heimark, L.; Pramanik, B.; Grilli, M.; Bertorelli, R.; Lozza, G.; Reggiani, A. Tricyclic thienopyridine-pyrimidones/thienopyrimidinepyrimidines as orally efficacious mGluR1 antagonists for neuropathic pain. Bioorg. Med. Chem. Lett. 2009, 19, 3199-3203.

10. Kim, Y.; Kim, J.; Kim, S.; Ki, Y.; Seo, S.H.; Tae, J.; Ko, M.K.; Jang, H.S.; Lim, E.J.; Song, C.; et al. Novel thienopyrimidinones as mGluR1 antagonists. Eur. J. Med. Chem. 2014, 85, 629-637.

11. Fundytus, M.E.; Yashpal, K.; Chabot, J.G.; Osborne, M.G.; Lefebvre, C.D.; Dray, A.; Henry, J.L.; Coderre, T.J. Knockdown of spinal metabotropic glutamate receptor $1\left(\mathrm{mGluR}_{1}\right)$ alleviates pain and restores opioid efficacy after nerve injury in rats. Br. J. Pharmacol. 2001, 132, 354-367.

Sample Availability: Samples of the compounds are not available from the authors.

(C) 2015 by the authors; licensee MDPI, Basel, Switzerland. This article is an open access article distributed under the terms and conditions of the Creative Commons Attribution license (http://creativecommons.org/licenses/by/4.0/). 